Report

\title{
A method for the generation of pseudotyped virus particles bearing SARS coronavirus spike protein in high yields
}

Yoichiro Fujioka, ${ }^{1,2, *}$ Sayaka Kashiwagi, ${ }^{1,2}$ Aiko Yoshida ${ }^{1,2}$, Aya O. Satoh, ${ }^{1}$ Mari Fujioka, ${ }^{1}$ Maho Amano ${ }^{1}$, Yohei Yamauchi ${ }^{3}$, and Yusuke Ohba ${ }^{1,2,4^{*}}$

${ }^{1}$ Department of Cell Physiology, Faculty of Medicine and Graduate School of Medicine, Hokkaido University, Sapporo 060-8638, Japan

${ }^{2}$ Global Station for Biosurfaces and Drug Discovery, Hokkaido University, Sapporo 0608612, Japan

${ }^{3}$ School of Cellular and Molecular Medicine, University of Bristol, University Walk, Bristol BS8 1TD, UK

${ }^{4}$ Lead Contact

*Correspondence: y-fuji@med.hokudai.ac.jp, yohba@med.hokudai.ac.jp

Department of Cell Physiology, Faculty of Medicine and Graduate School of Medicine, Hokkaido University, N15W7, Kita-ku, Sapporo 060-8638, Japan.

phone: +81-11-706-5158; fax: +81-11-706-7877 


\section{SUMMARY}

2 The ongoing severe acute respiratory syndrome coronavirus 2 (SARS-CoV-2) pandemic

3 has threatened human health and the global economy. Development of additional

4 vaccines and therapeutics is urgently required, but such development with live virus

5 must be conducted with biosafety level 3 confinement. Pseudotyped viruses have been

6 widely adopted for studies of virus entry and pharmaceutical development to overcome

7 this restriction. Here we describe a modified protocol to generate vesicular stomatitis

8 virus (VSV) pseudotyped with SARS-CoV or SARS-CoV-2 Spike protein in high yield.

9 We found that pseudovirions produced with the conventional transient expression

10 system lacked coronavirus Spike protein at their surface as a result of inhibition of

11 parental VSV infection by overexpression of this protein. Establishment of stable cell

12 lines with an optimal expression level of coronavirus Spike protein allowed the efficient

13 production of progeny pseudoviruses decorated with Spike protein. This improved VSV

14 pseudovirus production method should facilitate studies of coronavirus entry and

15 development of antiviral agents.

17 Keywords: severe acute respiratory syndrome coronavirus (SARS-CoV), SARS-CoV-2,

18 pseudovirus, vesicular stomatitis virus (VSV), Spike protein 


\section{INTRODUCTION}

2 Severe acute respiratory syndrome coronavirus 2 (SARS-CoV-2) is an enveloped, positive-

3 strand RNA virus that belongs to the Coronaviridae family and is responsible for the recent

4 pandemic of coronavirus disease 2019 (COVID-19). As of 15 June 2021, there had been $>174$

5 million confirmed cases of and $\sim 3.7$ million deaths from COVID-19 reported in over 220

6 countries, areas, or territories (https://www.who.int/emergencies/diseases/novel-coronavirus-

7 2019).

The envelope of SARS-CoV-2, like that of SARS-CoV, contains three structural

9 proteins: the spike glycoprotein (hereafter referred to simply as S protein), membrane protein,

10 and small envelope protein (Schoeman and Fielding, 2019; Wrapp et al., 2020). Together with

11 retrovirus envelope proteins and influenza virus hemagglutinin proteins, $S$ protein is

12 categorized as a class I viral fusion protein (Millet and Whittaker, 2018; White 2008), and it is

13 targeted to the rough endoplasmic reticulum ( $\mathrm{rER}$ ) of host cells by an $\mathrm{NH}_{2}$-terminal signal

14 sequence (Breitling and Aebi, 2013; Duan et al., 2020; Lontok et al., 2004; Sadasivan et al.,

2017). Cleavage of the signal sequence is followed by resumption of peptide chain elongation and insertion of the synthesized S protein into the ER membrane as a homotrimer (Binns et al., 1985; Braakman and Hebert, 2013; Delmas and Laude, 1990; Duan et al., 2020). S protein plays a key role in both specific interaction of the virus with its host cell receptor and subsequent virus internalization via endocytosis or membrane fusion. Binding of the coronavirus to its host cell receptor, angiotensin-converting enzyme 2 (ACE2), has been attributed to the $\mathrm{S} 1$ receptor-binding subunit region of $\mathrm{S}$ protein, which adopts an active "standing-up" conformation for receptor binding and an inactive "lying-down" conformation for immune evasion (Guan et al., 2020; Wrapp et al., 2020). After the S1 subunit region binds to ACE2, S protein undergoes proteolytic cleavage into $S 1$ and $S 2$ fusion subunits at the $\mathrm{S} 1 / \mathrm{S} 2$ site in the presence of transmembrane protease serine 2 (TMPRSS2) and furin (Bestle et al., 2020; Jaimes et al., 2020). Additional cleavage sites that are located within the S2 subunit and are cleaved by host cell proteases including cathepsins, furin-like proprotein convertases, and trypsin-like serine protease (Burkard et al., 2014; Coutard et al., 2020; Hoffmann et al., 2020). The exposed S2 subunit mediates membrane fusion either at the plasma membrane or with the endosomal membrane, depending on protease availability in the host cell. Characterization of S protein is thus crucial for a better understanding of SARS- 
$1 \quad$ Pseudotyped virus systems are highly useful for characterization of viral envelope 2 proteins and investigation of their role in virus entry (Hoffmann et al., 2020; Miyauchi et al.,

3 2009; Nanbo et al., 2010; Rentsch and Zimmer, 2011; Schelhaas et al., 2012; de Vries et al.,

4 2011). Virus particles produced with such a system consist of a surrogate viral core and

5 heterologous viral envelope proteins. The genome of the parental virus is modified to remove

6 essential genes for viral reproduction, so as to prevent the generation of infectious progeny

7 viruses. This approach allows viruses pseudotyped with envelope proteins of highly

8 pathogenic viruses to be handled safely in a BSL-2 containment facility instead of a BSL-3 or

9 BSL-4 facility. Vesicular stomatitis virus (VSV), a negative-strand RNA virus of the

10 Rhabdoviridae family, has been widely adopted in both pseudotyped and recombinant virus

11 systems (Hoffmann et al., 2020; Miyauchi et al., 2009; Nanbo et al., 2010; Rentsch and

12 Zimmer, 2011). It possesses five structural proteins- the glycoprotein (G), large polymerase

13 protein $(\mathrm{L})$, matrix protein $(\mathrm{M})$, nucleocapsid $(\mathrm{N})$, and phosphoprotein $(\mathrm{P})$ (Lichty et al.,

14 2004) — among which G protein contributes to binding to the host cell surface and fusion with

15 the endosomal membrane. VSV was the first negative-strand RNA virus for which a reverse

16 genetics approach was established (Lawson et al., 1995), an approach that allowed packaging

17 of a reporter gene into the viral genome for quantitative evaluation of infectivity of

18 recombinant viruses on the basis of the reporter gene activity. It was also found that a large

19 amount of morphologically intact virus particles could be produced even in the absence of G

20 protein. In addition, coinfection of cells with VSV and other viruses results in the formation

21 of VSV pseudotyped with heterologous envelope proteins (Huang et al., 1974; Witte and

22 Baltimore, 1977). With these features, VSV-based pseudotyping is a potentially powerful tool

23 for studies of the role of envelope proteins, including S proteins of SARS-CoV and SARS-

24 CoV-2, in virus entry (Clausen et al., 2020; Hoffmann et al., 2020).

25 During the course of experiments with VSV pseudoviruses bearing S protein of

26 SARS-CoV or SARS CoV-2 described here, we found that most pseudotyped particles

27 produced by the conventional transfection protocol lack $\mathrm{S}$ protein, likely because $\mathrm{S}$ protein

28 expression at a high level in the virus-producing cells suppresses infection by parental VSV.

29 We therefore developed an improved protocol based on cell lines stably expressing S proteins.

30 This new method generates VSV pseudotyped particles decorated with S protein at high

31 efficiency and with high infectious yields compared to the conventional approach. Our

32 protocol increases the fidelity of SARS-CoV-2 entry research and should facilitate the

33 development of new antiviral agents. 


\section{RESULTS AND DISCUSSION}

2 We initially aimed to evaluate the loading efficiency of S proteins on VSV pseudotyped virus

3 particles. According to the published protocol (Rentsch and Zimmer, 2011), HEK293T cells

4 transiently expressing SARS-CoV S protein were exposed to VSV $\Delta \mathrm{G}$ bearing $\mathrm{G}$ protein of

5 VSV (VSV $\Delta \mathrm{G}-\mathrm{G}$ ), and the resulting culture supernatant was harvested as a pseudovirus suspension in the presence of neutralizing antibodies to VSV G protein (to eliminate parental VSV $\Delta \mathrm{G}-\mathrm{G})$. The pseudotyped virions obtained in this manner were stained with the lipophilic carbocyanine dye DiI and then subjected to immunofluorescence analysis with a rabbit monoclonal antibody to SARS-CoV S protein. Confocal fluorescence imaging revealed that only a small proportion of the obtained particles harbored S protein (Figure 1A).

To examine $\mathrm{S}$ protein expression in the pseudotyped virion-producing cells, we prepared an expression vector for a fluorescent protein-tagged form of the protein. The coding sequence for mCherry, a red fluorescent protein (RFP), was thus inserted upstream of the $\mathrm{NH}_{2}$-terminal signal sequence of $\mathrm{S}$ protein, with the expectation that the fluorescent tag would be cleaved together with the signal sequence in the ER and so would not suppress virion formation. Indeed, immunoblot analysis of HEK293T cells transfected with the vector for mCherry-tagged S protein revealed separate fragments corresponding to mCherry and to $\mathrm{S}$ protein (Figure S1A), and no red fluorescence signal was detected from the VSV $\Delta$ GmCherry-S pseudoparticles produced by these cells (Figure S1B). Furthermore, determination of focus-forming units (FFU) by counting the number of cells positive for green fluorescent protein (GFP) encoded by the reporter gene packaged in the genome of parental VSV $\Delta \mathrm{G}$ (Rentsch and Zimmer, 2011) did not reveal a marked difference in infectivity between VSV $\Delta$ G-mCherry-S and VSV $\Delta$ G-S pseudoviruses in BEAS-2B cells expressing ACE2, also a receptor for SARS-CoV (Figure S1C). This result also supported the notion that the chimeric protein undergoes proteolytic cleavage and does not inhibit particle formation.

Fluorescence imaging of HEK293T cells transfected with expression vectors for mCherry-S or mCherry showed that mCherry-S expression interfered with VSV $\Delta \mathrm{G}-\mathrm{G}$ infection; the GFP signal was thus detected in cells with no or only a low level of mCherry-S

30 expression but not in highly red fluorescent cells (Figure 1B). Quantitative analysis revealed that GFP-positive cells were indeed highly enriched in cells in which red fluorescence intensity was $<10,000$ arbitrary units (A.U.) (Figure 1C), whereas a high level of mCherry 
1 also suggested that virus particles lacking envelope protein might be produced from cells with

2 no mCherry-S expression. Indeed, immunofluorescence analysis with antibodies to VSV M

3 protein showed that the supernatant of vector-transfected, VSV $\Delta$ G-G-exposed HEK293T

4 cells contained fluorescent puncta even when the vector did not encode an envelope protein

5 (Figure S1E).

Our results together suggested that a low and persistent level of $\mathrm{S}$ protein expression

7 might be required for the production of pseudotyped virus particles that uniformly bear $\mathrm{S}$

8 protein. We therefore attempted to establish cell lines that stably express S protein. However,

9 although HEK293T cells are used in the standard protocol for VSV-based pseudotyped virus

10 production, we found that HEK293T cells expressing mCherry-S manifested a shrunken

11 morphology compared with those expressing mCherry alone (Figure S1F). We next

12 introduced the expression vector for mCherry-S into VeroE6 cells, which have been widely

13 adopted for SARS-CoV research. Fluorescence imaging showed that the morphology of

14 VeroE6 cells expressing mCherry-S was similar to that of those expressing mCherry alone

15 (Figure 2A). To generate stable cell lines, we isolated transfected VeroE6 cells by treatment

16 with trypsin and subjected them to puromycin selection. After incubation for $24 \mathrm{~h}$,

17 multinucleated cells, indicative of cell-cell fusion resulting from $\mathrm{S}$ protein activation by

18 trypsin cleavage, were apparent for the cells expressing mCherry-S (Figure 2B). To avoid

19 cleavage of S protein, we used enzyme-free cell-dissociation solutions for cell isolation.

20 Treatment with such solutions did not induce cleavage of S protein (Figure S2A) or

21 multinucleated cells (Figure 2C), thus allowing the generation of cell lines stably expressing

22 S protein (Figure 2D).

23 We next examined whether such a cell line was susceptible to VSV $\Delta$ G-G infection for 24 production of pseudoviruses harboring S protein. Fluorescence microscopy and quantitative 25 analysis of the virus-exposed cells revealed that the mCherry fluorescence intensity of all 26 cells was $<10,000$ A.U. (the desired upper limit as determined in Figure 1C), and that most 27 cells were positive for GFP, indicative of uniform VSV $\Delta$ G-G infection (Figures S2B and 28 S2C). The virus particles produced by the cells were then stained with DiD and adsorbed on a polyethylenimine-coated glass-based dish to determine the amount of particles per unit volume by measurement of the area of DiD-positive puncta with a confocal microscope. The amount of particles produced by VeroE6 cells stably expressing mCherry-S was $\sim 10 \%$ of that

32 of those produced by HEK293T cells transiently expressing mCherry-S (Figures S3A and 33 S3B). BEAS-2B cells expressing ACE2 were then exposed to the pseudoviruses for $1 \mathrm{~h}$ at $34 \quad 4^{\circ} \mathrm{C}$ (to allow for virus attachment) and subjected to immunofluorescence analysis with 
1 antibodies to SARS-CoV S protein and to VSV M protein in order to allow visualization of S

2 protein and all pseudovirus particles, respectively. Confocal imaging showed that, in contrast

3 to the virus particles produced by transiently transfected HEK293T cells, essentially all virus

4 particles produced by the stably transfected VeroE6 cell line were positive for S protein

5 (Figure 3A). Indeed, quantitative analysis revealed that the fraction of S protein-positive

6 particles generated by the stable cell line was about seven times that generated by the

7 transiently transfected cells (Figure 3B). These results thus demonstrated the efficient

8 production of pseudoviruses that were essentially all loaded with S protein by the stable cell

9 line.

10 We further examined whether the titer of pseudovirus particles generated by the stable 11 cell line was higher than that of particles generated by the conventional method. There was no 12 significant difference between the FFU values obtained for the two types of pseudoviruses

13 (Figure S3C). To normalize infectivity, we prepared dilutions of the virus suspensions (down 14 to $1.0 \mathrm{FFU} / \mu \mathrm{l})$ and plotted FFU against the area of puncta. A linear relation was apparent for 15 each type of pseudovirus, although the slopes of the regression lines differed (Figure S3D).

16 The FFU value normalized by the amount of viruses produced by the stable cell line was 6.4

17 times the corresponding value for the viruses produced by the transiently transfected cells

18 (Figure 3C), indicating that the modified method established in this study results in a

19 substantial improvement in infectious pseudovirus particle production.

Finally, we attempted to produce pseudotyped virus particles with envelopes bearing the S protein of SARS-CoV-2 by our newly established protocol. We thus established a VeroE6 cell line that stably expresses SARS-CoV-2 S protein (Figure S4A). The fraction of SARS-CoV-2 S protein-positive puncta (Figures 4A and 4B) and FFU normalized by the area of DiD-positive puncta (Figures 4C, S4B and S4C) were five and seven times as high, respectively, for viruses produced from the VeroE6 cell line as were those for viruses produced from transiently transfected HEK293T cells. Our procedure was thus shown to be highly efficient with regard to pseudovirus production for studies of the entry of SARS-CoV or SARS-CoV-2.

In this study, we unexpectedly found that a large proportion of pseudotyped virus particles produced by the conventional protocol (Hoffmann et al., 2020; Rentsch and Zimmer, 2011) did not bear coronavirus $S$ proteins. This finding raised the concern that $S$ protein-free 32 virions might affect the entry process of, or cellular responses to, $\mathrm{S}$ protein-bearing particles 33 and thereby confound experimental outcomes. We therefore established a modified protocol 34 based on stable cell lines in order to generate pseudoviruses for which a large proportion of 
1 the virus particles harbor heterologous envelope proteins. Alternative methods that are free of

2 such issues relating to virus particles lacking envelope protein, including reverse genetic

3 systems for in vitro virus assembly from SARS-CoV-2 full-length cDNA (Torii et al., 2021;

4 Xie et al., 2020), have been developed. However, given that the VSV-based pseudotyped

5 virus systems have been adopted by many researchers, the modification described here should

6 be readily incorporated into current protocols and lead both to a better understanding of virus

7 entry and to accelerated vaccine and therapeutic development for SARS-CoV-2.

\section{ACKNOWLEDGEMENTS}

10 We thank Dr. S. Pöhlmann for expression plasmids, viruses and cells, as well as A. Kikuchi

11 for technical assistance. This work was supported in part by Grants-in-Aid from the Ministry

12 of Education, Culture, Sports, Science and Technology of Japan (\#26115701 and \#15H01248),

13 from the Japan Society for the Promotion of Science (\#19H05411, \#20H05872, \#21H02735

14 and \#21H00413), and from the Japan Agency for Medical Research and Development

15 (JP17fk0108124j0601), as well as by COVID-19 Drug and Vaccine Development Donation.

17 AUTHOR CONTRIBUTIONS

18 Conceptualization, Y.F. and Y.O.; Methodology, Y.F. and S.K.; Formal analysis, Y.F. and 19 S.K.; Investigation, Y.F., S.K., A.Y., A.O.S., and M.F.; Resources, Y.F., S.K., A.Y., A.O.S., 20 and M.F.; Writing-original draft, Y.F. and Y.O.; Writing-review and editing, Y.F., M.A.,

21 Y.Y., and Y.O.; Visualization, Y.F. and Y.O.; Supervision, Y.Y. and Y.O.; Project

22 administration, Y.O.; Funding acquisition, Y.F. and Y.O.

\section{DECLARATION OF INTERESTS}

25 Y.F. and Y.O have a patent pending that includes claims relating to the present study. Other authors declare no competing interests. 


\section{REFERENCES}

2 Bestle, D., Heindl, M.R., Limburg, H., van Lam van, T., Pilgram, O., Moulton, H., Stein,

3 D.A., Hardes, K., Eickmann, M., Dolnik, O., et al. (2020). TMPRSS2 and furin are both

4 essential for proteolytic activation of SARS-CoV-2 in human airway cells. Life Sci. Alliance

53 , e202000786.

6 Binns, M.M., Boursnell, M.E.G., Cavanagh, D., Pappin, D.J., and Brown, T.D. (1985).

7 Cloning and sequencing of the gene encoding the spike protein of the coronavirus IBV. J. Gen.

8 Virol. 66, 719-726.

9 Braakman, I., and Hebert, D.N. (2013). Protein folding in the endoplasmic reticulum. Cold

10 Spring Harb. Perspect. Biol. 5, a013201.

11 Breitling, J., and Aebi, M. (2013). N-linked protein glycosylation in the endoplasmic

12 reticulum. Cold Spring Harb. Perspect. Biol. 5, a013359.

Burkard, C., Verheije, M.H., Wicht, O., van Kasteren, S.I., van Kuppeveld, F.J., Haagmans, B.L., Pelkmans, L., Rottier, P.J.M., Bosch, B.J., and de Haan, C.A.M. (2014). Coronavirus Cell Entry Occurs through the Endo-/Lysosomal Pathway in a Proteolysis-Dependent Manner. PLOS Pathog. 10, e1004502.

Clausen, T.M., Sandoval, D.R., Spliid, C.B., Pihl, J., Perrett, H.R., Painter, C.D., Narayanan, A., Majowicz, S.A., Kwong, E.M., McVicar, R.N., et al. (2020). SARS-CoV-2 Infection Depends on Cellular Heparan Sulfate and ACE2. Cell 183, 1043-1057.

Coutard, B., Valle, C., de Lamballerie, X., Canard, B., Seidah, N.G., and Decroly, E. (2020). The spike glycoprotein of the new coronavirus 2019-nCoV contains a furin-like cleavage site absent in CoV of the same clade. Antiviral Res. 176, 104742.

Daly, J.L., Simonetti, B., Klein, K., Chen, K.E., Williamson, M.K., Antón-Plágaro, C., Shoemark, D.K., Simón-Gracia, L., Bauer, M., Hollandi, R., et al. (2020). Neuropilin-1 is a host factor for SARS-CoV-2 infection. Science 370, 861-865.

Delmas, B., and Laude, H. (1990). Assembly of coronavirus spike protein into trimers and its 27 role in epitope expression. J. Virol. 64, 5367-5375.

Duan, L., Zheng, Q., Zhang, H., Niu, Y., Lou, Y., and Wang, H. (2020). The SARS-CoV-2 Spike Glycoprotein Biosynthesis, Structure, Function, and Antigenicity: Implications for the 30 Design of Spike-Based Vaccine Immunogens. Front. Immunol. 11, 2593.

Fujioka, Y., Nishide, S., Ose, T., Suzuki, T., Kato, I., Fukuhara, H., Fujioka, M., Horiuchi, K., Satoh, A.O., Nepal, P., et al. (2018). A Sialylated Voltage-Dependent $\mathrm{Ca}^{2+}$ Channel Binds Hemagglutinin and Mediates Influenza A Virus Entry into Mammalian Cells. Cell Host Microbe 23, 809-818.

Fujioka, Y., Satoh, A.O., Horiuchi, K., Fujioka, M., Tsutsumi, K., Sasaki, J., Nepal, P.,

36 Kashiwagi, S., Paudel, S., Nishide, S., et al. (2019). A Peptide Derived from Phosphoinositide

37 3-kinase Inhibits Endocytosis and Influenza Virus Infection. Cell Struct. Funct. 44, 61-74. 
1 Syndrome Coronavirus Spike Glycoprotein Provides Insights into Evolution of Unique

2 Coronavirus Spike Proteins. J. Virol. 94, e01301-20.

3 Hoffmann, M., Kleine-Weber, H., Schroeder, S., Krüger, N., Herrler, T., Erichsen, S.,

4 Schiergens, T.S., Herrler, G., Wu, N.H., Nitsche, A., et al. (2020). SARS-CoV-2 Cell Entry

5 Depends on ACE2 and TMPRSS2 and Is Blocked by a Clinically Proven Protease Inhibitor.

6 Cell 181,271-280.e8.

7 Huang, A.S., Palma, E.L., Hewlett, N., and Roizman, B. (1974). Pseudotype formation

8 between enveloped RNA and DNA viruses. Nature 252, 743-745.

9 Jaimes, J.A., Millet, J.K., and Whittaker, G.R. (2020). Proteolytic Cleavage of the SARS-

10 CoV-2 Spike Protein and the Role of the Novel S1/S2 Site. iScience 23, 101212.

11 Kashiwagi, S., Fujioka, Y., Satoh, A.O., Yoshida, A., and Fujioka, M. (2019a). Folding 12 latency of fluorescent proteins affects the mitochondrial localization of fusion proteins. Cell

13 Struct. Funct. 44, 183-194.

14 Kashiwagi, S., Fujioka, Y., Kondo, T., Satoh, A.O., and Yoshida, A. (2019b). Localization of 15 BCR-ABL to stress granules contributes to its oncogenic function. Cell Struct. Funct. 44, 16 195-204.

17 Lawson, N.D., Stillman, E.A., Whitt, M.A., and Rose, J.K. (1995). Recombinant vesicular 18 stomatitis viruses from DNA. Proc. Natl. Acad. Sci. USA 92, 4477-4481.

19 Lichty, B.D., Power, A.T., Stojdl, D.F., and Bell, J.C. (2004). Vesicular stomatitis virus: Re20 inventing the bullet. Trends Mol. Med. 10, 210-216.

21 Lontok, E., Corse, E., and Machamer, C.E. (2004). Intracellular Targeting Signals Contribute 22 to Localization of Coronavirus Spike Proteins near the Virus Assembly Site. J. Virol. 78, $23 \quad 5913-5922$.

24 Millet, J.K., and Whittaker, G.R. (2018). Physiological and molecular triggers for SARS-CoV 25 membrane fusion and entry into host cells. Virology 517, 3-8.

26 Miyauchi, K., Kim, Y., Latinovic, O., Morozov, V., and Melikyan, G.B. (2009). HIV Enters

27 Cells via Endocytosis and Dynamin-Dependent Fusion with Endosomes. Cell 137, 433-444.

28 Miyoshi, H., Blömer, U., Takahashi, M., Gage, F.H., and Verma, I.M. (1998). Development 29 of a Self-Inactivating Lentivirus Vector. J. Virol. 72, 8150-8157.

30 Nanbo, A., Imai, M., Watanabe, S., Noda, T., Takahashi, K., Neumann, G., Halfmann, P., and 31 Kawaoka, Y. (2010). Ebolavirus is internalized into host cells via macropinocytosis in a viral 32 glycoprotein-dependent manner. PLOS Pathog. 6, e1001121.

33 Rentsch, M.B., and Zimmer, G. (2011). A vesicular stomatitis virus replicon-based bioassay 34 for the rapid and sensitive determination of multi-species type I interferon. PLOS ONE 6, 35 e25858.

36 Sadasivan, J., Singh, M., and Sarma, J. Das (2017). Cytoplasmic tail of coronavirus spike 37 protein has intracellular targeting signals. J. Biosci. 42, 231-244. 
1 Schelhaas, M., Shah, B., Holzer, M., Blattmann, P., Kühling, L., Day, P.M., Schiller, J.T., and 2 Helenius, A. (2012). Entry of human papillomavirus type 16 by actin-dependent, clathrin- and 3 lipid raft-independent endocytosis. PLOS Pathog. 8, e1002657.

4 Schoeman, D., and Fielding, B.C. (2019). Coronavirus envelope protein: Current knowledge. 5 Virol. J. 16, 1-22.

6 Torii, S., Ono, C., Suzuki, R., Morioka, Y., Anzai, I., Fauzyah, Y., Maeda, Y., Kamitani, W., 7 Fukuhara, T., and Matsuura, Y. (2021). Establishment of a reverse genetics system for SARS8 CoV-2 using circular polymerase extension reaction. Cell Rep. 35, 109014.

9 de Vries, E., Tscherne, D.M., Wienholts, M.J., Cobos-Jiménez, V., Scholte, F., García-Sastre, 10 A., Rottier, P.J.M., and de Haan, C.A.M. (2011). Dissection of the influenza a virus endocytic 11 routes reveals macropinocytosis as an alternative entry pathway. PLOS Pathog. 7, e1001329.

12 Witte, O.N., and Baltimore, D. (1977). Mechanism of formation of pseudotypes between 13 vesicular stomatitis virus and murine leukemia virus. Cell 11, 505-511.

14 Wrapp, D., Wang, N., Corbett, K.S., Goldsmith, J.A., Hsieh, C.-L., Abiona, O., Graham, B.S., 15 and McLellan, J.S. (2020). Cryo-EM structure of the 2019-nCoV spike in the prefusion 16 conformation. Science $367,1260-1263$.

17 Xie, X., Muruato, A., Lokugamage, K.G., Narayanan, K., Zhang, X., Zou, J., Liu, J., 18 Schindewolf, C., Bopp, N.E., Aguilar, P. V., et al. (2020). An Infectious cDNA Clone of 19 SARS-CoV-2. Cell Host Microbe 27, 841-848.e3. 


\section{STAR Methods}

\section{Contact for reagent and resource sharing}

3 Further information and requests for resources and reagents should be directed to and will be

4 fulfilled by the Lead Contact, Yusuke Ohba (yohba@med.hokudai.ac.jp).

\section{$5 \quad$ Cell lines}

6 HEK293T (ATCC CRL-11268), VeroE6 (CRL-1586), and BEAS-2B (CRL-9609) cells were

7 obtained from American Type Culture Collection (ATCC; Manassas, VA) and were

8 maintained under a humidified atmosphere of $5 \% \mathrm{CO}_{2}$ at $37^{\circ} \mathrm{C}$ in Dulbecco's modified

9 Eagle's medium (DMEM) (Sigma-Aldrich, Tokyo, Japan) supplemented with $10 \%$ fetal bovine serum (FBS) (Capricorn Scientific, Ebsdorfergrund, Germany). BHK21/G43 were kindly provided by S. Pöhlmann (Leibniz Institute for Primate Research, Göttingen,

12 Germany). Expression plasmids were introduced into cells by transfection for $24 \mathrm{~h}$ with the 13 use of Polyethylenimine "Max" (PEI MAX; Polysciences, Warrington, PA). The absence of 14 mycoplasma contamination was confirmed with the use of a PCR Mycoplasma Test Kit 15 (Takara, Shiga, Japan).

\section{Reagents and antibodies}

17 Antibodies to SARS-CoV S protein (\#40150-R007), to RFP (\#PM005), to VSV G protein (\#EB0010), and to VSV M protein (\#MABF2347) were obtained from Sino Biological (Beijing, China), Medical \& Biological Laboratory (Nagoya, Japan), Kerafast (Boston, MA), and Merck (Darmstadt, Germany), respectively. Hoechst 33342, carbocyanine dyes (DiO, DiI, and DiD), and Alexa Fluor 647- or Alexa Fluor 488-labeled antibodies to rabbit or mouse immunoglobulin G were obtained from Thermo Fisher Scientific (Carlsbad, CA). Horseradish peroxidase (HRP)-conjugated goat secondary antibodies were obtained from Jackson

24 ImmunoResearch Laboratories (West Grove, PA). Trypsin was obtained from FujiFilm-Wako

25 (Osaka, Japan), and cell-dissociation solutions were from Thermo Fisher Scientific and

Biological Industries (Beit-Haemek, Israel). Mifepristone was obtained from Sigma-Aldrich.

\section{Plasmids}

28 Expression vectors for the S protein of SARS-CoV or SARS-CoV-2 (pCG1-SARS-CoV-S and pCG1-SARS-CoV-2-S) (Hoffmann et al., 2020) were kindly provided by S. Pöhlmann.

30 The coding sequence for mCherry was cleaved out of pFX-Tom20-mCherry (Kashiwagi et al., 312019 a) by digestion with BamHI and BglII, and was then subcloned into the BamHI sites of 32 the expression vectors for the S proteins to obtain pCG1-mCherry-SARS-CoV-S and pCG1- 
1 mCherry-SARS-CoV-2-S. The pBABE-puro plasmid encoding a puromycin resistance gene

2 was obtained from Addgene.

\section{Generation of stable cell lines}

4 pCG1-mCherry-SARS-CoV-S or pCG1-mCherry-SARS-CoV-2-S was linearized with PuvI

5 and introduced together with pBABE-puro into HEK293T or VeroE6 cells by transfection for

$624 \mathrm{~h}$ with the use of PEI MAX. The cells were then cultured in DMEM supplemented with

$7 \quad 10 \%$ FBS and puromycin $(10 \mu \mathrm{g} / \mathrm{ml})$, and puromycin-resistant colonies were collectively

8 harvested. Expression of the mCherry-tagged $\mathrm{S}$ proteins was confirmed by

9 immunofluorescence analysis.

BEAS-2B cells stably expressing human ACE2 were generated by lentivirus-mediated gene transfer. HEK293T cells were thus transfected with pLVX-ACE2-IRES-BLD (Daly et al., 2020), pCAG-HIVgp, and pCMV-VSVG-RSV-Rev (Miyoshi et al., 1998) for 48 h, after

which the culture supernatant was collected. BEAS-2B cells were exposed to the recombinant lentivirus-containing supernatant for $1 \mathrm{~h}$ at $35^{\circ} \mathrm{C}$, cultured for 2 days at $35^{\circ} \mathrm{C}$ in DMEM supplemented with 10\% FBS, and further cultured for 14 days in DMEM supplemented with 10\% FBS and blasticidin $(20 \mu \mathrm{g} / \mathrm{ml})$ (Funakoshi, Tokyo, Japan). The resulting blasticidinresistant colonies were collectively harvested, and the constituent cells were maintained in DMEM supplemented with $10 \%$ FBS and blasticidin $(15 \mu \mathrm{g} / \mathrm{ml})$.

\section{Preparation of VSV pseudoviruses}

20 Pseudotyped viruses were generated according to an established protocol (Rentsch and 21 Zimmer, 2011) or its modified version developed in the present study. In brief, for preparation of VSV $\triangle \mathrm{G}-\mathrm{G}, \mathrm{BHK} 21 / \mathrm{G} 43$ cells (which stably express VSV G protein) were incubated with mifepristone $(10 \mathrm{nM})$ for $6 \mathrm{~h}$ and then infected with VSV $\Delta \mathrm{G}$ at a multiplicity of infection (MOI) of $100,000 \mathrm{FFU}$ per cell for $16 \mathrm{~h}$ at $35^{\circ} \mathrm{C}$. For generation of VSV $\Delta \mathrm{G}-\mathrm{S}$ particles, cells expressing S protein of SARS-CoV or SARS-CoV-2 (either stably or transiently) were exposed to VSV $\Delta \mathrm{G}-\mathrm{G}$ at an MOI of $3 \mathrm{FFU}$ per cell for $16 \mathrm{~h}$ at $35^{\circ} \mathrm{C}$ in the presence of antibodies to VSV G in order to neutralize the parental viruses. The culture supernatants were passed through a filter (pore size of $0.45 \mu \mathrm{m}$ ) and subjected to ultracentrifugation, and the virus pellets were resuspended in phenol red-free DMEM/F12 (Thermo Fisher Scientific) and stored at $-80^{\circ} \mathrm{C}$ until use. Given that the enhanced GFP (EGFP) gene is packaged in the genome of the pseudoviruses in this system (Rentsch and Zimmer, 2011), the titer of 


\section{Determination of FFU}

2 BEAS-2B cells expressing ACE2 were incubated with pseudotyped viruses for $2 \mathrm{~h}$ at $35^{\circ} \mathrm{C}$,

3 and the culture supernatants were then removed and the cells cultured in DMEM for $16 \mathrm{~h}$ at

$435^{\circ} \mathrm{C}$ before staining with Hoechst 33342 for 15 min and examination with a fluorescence

5 microscope. The FFU value of the pseudovirus suspension was determined by counting the

6 number of GFP-positive cells with the use of the "Multi-wavelength cell scoring" module of

7 MetaMorph software (Molecular Devices, CA).

\section{Fluorescence microscopy}

9 Fluorescence imaging and data analysis were performed essentially as described previously

10 (Fujioka et al., 2019; Kashiwagi et al., 2019b). In brief, cells transfected with mCherry-CoV-

11 S or mCherry-CoV-2-S vectors were stained with Hoechst 33342 for 15 min and placed in a

12 stage-top incubation chamber maintained at $37^{\circ} \mathrm{C}$ on a Nikon Ti2 microscope (Nikon, Tokyo,

13 Japan) equipped with a Zyla5.5 scientific complementary metal oxide semiconductor camera

14 (Oxford Instruments, Belfast, UK). The cells were illuminated with an X-Cite turbo system

15 (Excelitas Technologies, Waltham, MA) through a GFP HQ, Cy3 HQ, or DAPI-U HQ

16 excitation filter (Nikon).

\section{Fluorescent labeling of pseudoviruses}

18 For preparation of fluorescently labeled pseudoviruses, $1 \mathrm{ml}$ of pseudovirus suspension was

19 incubated for $1 \mathrm{~h}$ at room temperature with $6 \mu \mathrm{l}$ of $100 \mu \mathrm{M} \mathrm{DiD}$, DiI, or DiO stock solution in

20 the dark (Fujioka et al., 2018; Nanbo et al., 2010). The stained particles were adsorbed to a

21 PEI MAX-coated 96-well glass-based plate and observed with a confocal microscope.

\section{Immunofluorescence analysis}

23 BEAS-2B cells expressing ACE2 were incubated with pseudotyped viruses for $1 \mathrm{~h}$ at $4^{\circ} \mathrm{C}$ to

allow for virus attachment. The cells were then fixed with 3\% paraformaldehyde for $15 \mathrm{~min}$ at

room temperature and incubated with $1 \%$ bovine serum albumin to block nonspecific binding

26 of antibodies. They were further incubated overnight at $4^{\circ} \mathrm{C}$ with primary antibodies $(1: 1000$

27 dilution), after which immune complexes were detected by incubation for $1 \mathrm{~h}$ at room

28 temperature in the dark with Alexa Fluor 488- or Alexa Fluor 647-conjugated secondary

29 antibodies (1:250 dilution). Nuclei were visualized by staining with Hoechst 33342. Images

30 were acquired with an IX83 microscope (Olympus, Tokyo, Japan) with X-lightV3

31 (CrestOptics, Rome, Italy). 


\section{Immunoblot analysis}

2 Transfected HEK293T cells were lysed in a lysis buffer [50 mM Tris- $\mathrm{HCl}$ (pH 7.4), $150 \mathrm{mM}$

$3 \mathrm{NaCl}, 1 \%$ Nonidet P-40, $0.5 \%$ sodium deoxycholate, $0.1 \%$ SDS, $1 \mathrm{mM} \mathrm{Na}_{3} \mathrm{VO}_{4}$, cOmplete

4 Protease Inhibitor Cocktail (Sigma-Aldrich)] for $30 \mathrm{~min}$ on ice. The lysates were centrifuged

5 at $20,000 \times g$ for $10 \mathrm{~min}$ at $4^{\circ} \mathrm{C}$, and the resulting supernatants were subjected to SDS-

6 polyacrylamide gel electrophoresis. The separated proteins were transferred to a

7 polyvinylidene difluoride membrane (Bio-Rad, Hercules, CA) and subjected to immunoblot

8 analysis. Immune complexes were detected with HRP-conjugated secondary antibodies, ECL

9 Western Blotting Detection Reagent (Cytiva, Tokyo, Japan) and a MIIS imaging system

10 (Givetechs, Sakura, Japan).

\section{Quantification and statistical analysis}

12 Quantitative data are presented as means + SEM from at least three independent experiments 13 and were compared with Student's $t$ test (parametric test between two conditions) or by one14 way analysis of variance (ANOVA) followed by the Tukey honestly significant difference 15 (HSD) post hoc test (among multiple conditions). No statistical methods were applied to 16 predetermine sample size. Experiments were performed unblinded. A $p$ value of $<0.05$ was

17 considered statistically significant, and all statistical analysis was performed with JMP Pro 18 software (version 15.0.0). 


\section{FIGURE LEGENDS}

Figure 1. Pseudotyped virus particles produced from cells transiently expressing SARSCoV S protein are not loaded with $S$ protein

(A) HEK293T cells were transfected with an expression vector for SARS-CoV S protein as well as infected with VSV $\Delta$ G-G. The culture supernatant was collected and stained with DiI as well as subjected to immunofluorescence staining with antibodies to SARS-CoV S protein and Alexa Fluor 488-conjugated secondary antibodies. The virus particles were then adsorbed onto a polyethylenimine-coated glass-based plate and observed with a confocal fluorescence microscope. Representative images are shown. Bars, $10 \mu \mathrm{m}$.

(B) HEK293T cells were transfected with an expression vector for mCherry-tagged SARSCoV S protein and infected with VSV $\Delta$ G-G for $16 \mathrm{~h}$. They were then observed with a fluorescence microscope for detection of mCherry (red) and GFP (green) fluorescence. Representative images are shown. The boxed region in the lower right panel is shown at higher magnification in the other panels. Bars, $10 \mu \mathrm{m}$.

(C) Fluorescence intensities of GFP and mCherry in individual cells as in (B). See also Figure S1.

Figure 2. Trypsin treatment induces cell-cell fusion of $\mathbf{S}$ protein-expressing cells (A) VeroE6 cells transfected with an expression vector for mCherry or mCherry-tagged $\mathrm{S}$ protein of SARS-CoV (mCherry-S) that had been linearized with PuvI were stained with Hoechst 33342 and observed by fluorescence microscopy. Representative images of mCherry (magenta) and Hoechst 33342 (blue) fluorescence are shown. Bars, $10 \mu \mathrm{m}$.

(B and C) Cells prepared as in (A) were harvested by exposure to trypsin (B) or to enzymefree cell dissociation solutions from Biological Industries (BI) or Gibco $(\mathbf{C})$. They were then transferred to cell culture dishes and cultured for $24 \mathrm{~h}$ before fluorescence microscopic analysis as in (A). Representative images are shown. Bars, $10 \mu \mathrm{m}$.

(D) Cells transfected with the mCherry-S vector (or the corresponding empty vector) as in (A) were cultured in the presence of puromycin for 14 days, stained with Hoechst 33342, and subjected to immunofluorescence analysis with antibodies to SARS-CoV S protein and Alexa Fluor 488-conjugated secondary antibodies. Representative fluorescence microscopic images of S protein immunostaining (green) as well as of mCherry (magenta) and Hoechst 33342 (blue) fluorescence are shown. Bars, $10 \mu \mathrm{m}$.

See also Figure $\mathbf{S 2}$.

Figure 3. A large proportion of pseudotyped virus particles produced from VeroE6 cells stably expressing SARS-CoV S protein are loaded with $S$ protein.

(A) VeroE6 cells stably expressing or HEK293T cells transiently expressing SARS-CoV S protein were infected with VSV $\Delta \mathrm{G}-\mathrm{G}$ for $16 \mathrm{~h}$, and equal numbers of pseudoviruses released into the culture supernatants were added to ACE2-expressing BEAS-2B cells for $1 \mathrm{~h}$ at $4^{\circ} \mathrm{C}$. The latter cells were then fixed, stained with Hoechst 33342 (blue), subjected to immunofluorescence analysis with antibodies to SARS-CoV S protein and VSV M protein, and observed with a confocal microscope. Representative images are shown. The boxed 
regions in the right panels are shown at higher magnification in the panels to the left. Bars, 10 $\mu \mathrm{m}$.

(B) Quantification of the fraction of S protein-positive puncta among all M protein-positive puncta for images as in (A). Data are means + SEM from three independent experiments. *, $p$ $<0.001$ (Student's $t$ test).

(C) BEAS-2B cells stably expressing ACE2 were exposed to pseudotyped viruses prepared as in (A) for determination of the number of GFP-positive cells and calculation of FFU of virus suspension. FFU was normalized by the amount of pseudotyped viruses, which was determined by the area of puncta of DiD-stained viruses on a polyethylenimine-coated glassbased plate. Data are means + SEM from three independent experiments. ${ }^{*}, p<0.004$ (oneway ANOVA with Tukey's HSD post hoc test).

See also Figure S3.

\section{Figure 4. Production of pseudoviruses loaded with S protein of SARS-CoV-2 by a stable cell line}

(A) BEAS-2B cells expressing ACE2 were exposed for $1 \mathrm{~h}$ at $4^{\circ} \mathrm{C}$ to pseudotyped viruses produced from a VeroE6 cell line stably expressing or HEK293T cells transiently expressing S protein of SARS-CoV-2. The cells were then fixed, stained with Hoechst 33342 (blue), subjected to immunofluorescence staining with antibodies to SARS-CoV S protein and VSV $M$ protein, and examined with a confocal microscope. Representative images are shown. The boxed regions in the bottom panels are shown at higher magnification in the panels above.

Bars, $10 \mu \mathrm{m}$.

(B) Quantification of the fraction of S protein-positive puncta among all M protein-positive puncta in images as in (A). Data are means + SEM from three independent experiments. ${ }^{*} p<$ 0.001 (Student's t test).

(C) FFU normalized by the area of puncta for the pseudoviruses produced as in (A) was determined as in Figure S4. Data are means + SEM from three independent experiments. *, $p$ $<0.004$ (one-way ANOVA with Tukey's HSD post-hoc test).

See also Figure $\mathbf{S 4}$. 
bioRxiv preprint doi: https://doi.org/10.1101/2021.07.30.454063; this version posted July 30, 2021. The copyright holder for this preprint (which

A was not certified by peer review) is the author/funder. All rights reserved. No reuse allowed without permission. Anti-SARS-CoV S (Alexa Fluor 488)

Dil

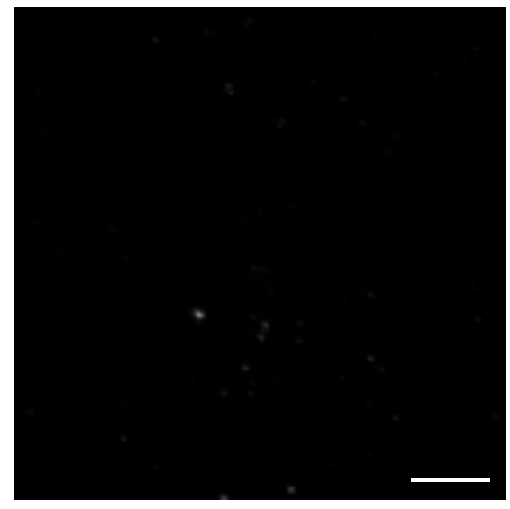

B

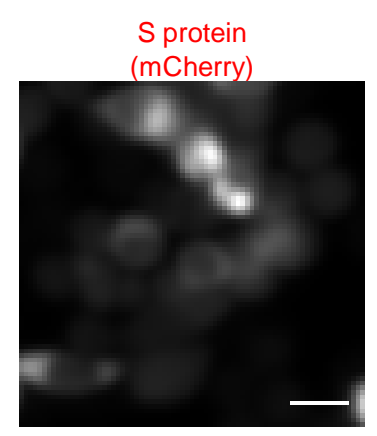

Merged

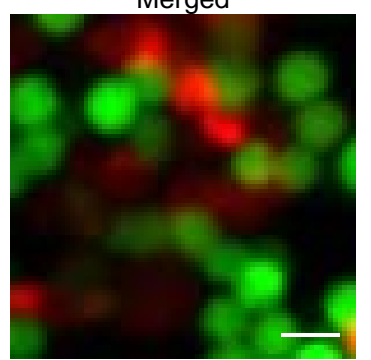

VSVAG-G

(EGFP)
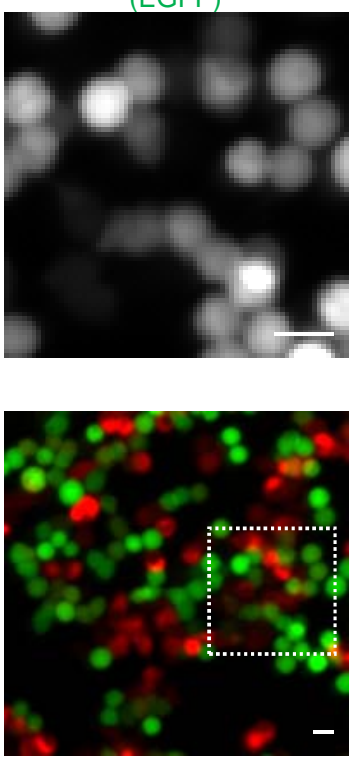

Merged

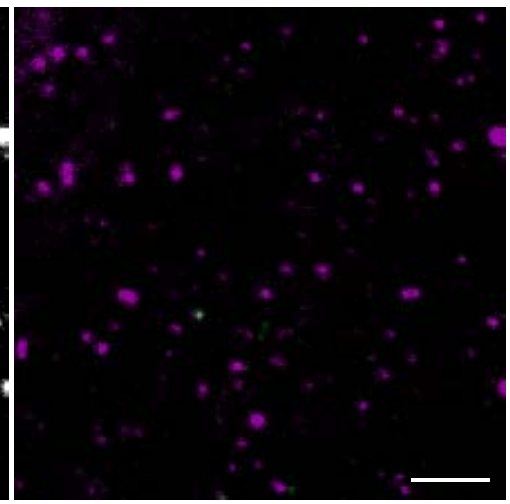

C

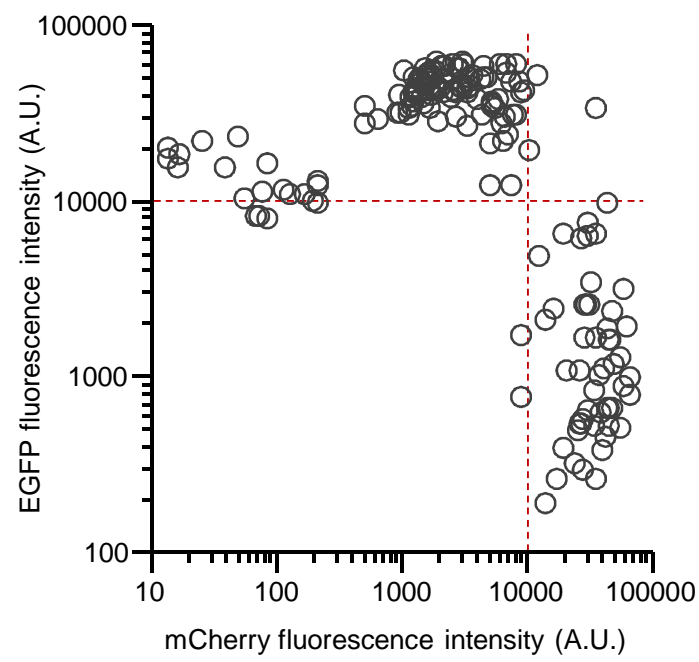


bioRxiv preprint doi: https://doi.org/10.1101/2021.07.30.454063; this version posted July 30, 2021. The copyright holder for this preprint (which was not certified by peer review) is the author/funder. All rights reserved. No reuse allowed without permission.

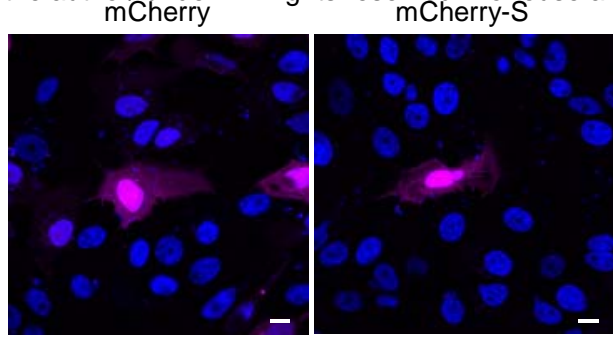

B

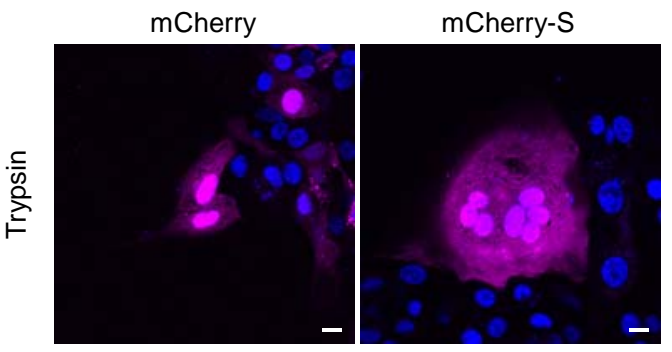

C

mCherry

mCherry-S

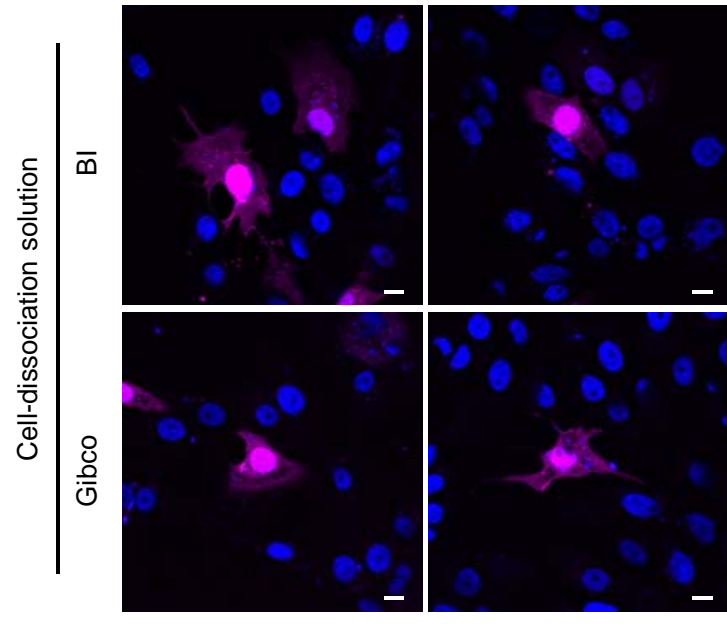

D

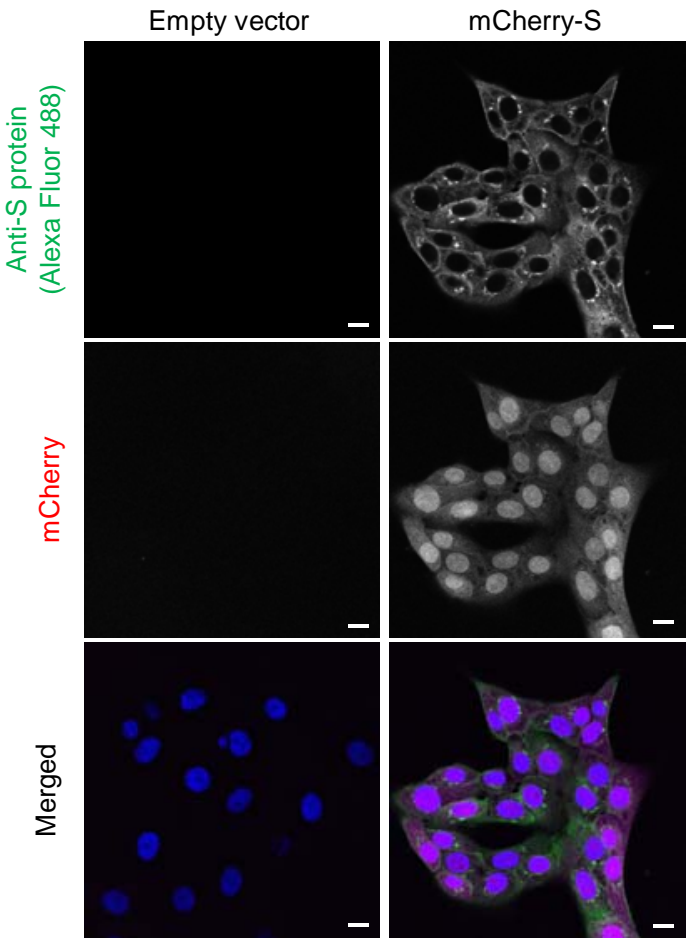

Y. Fujioka, et al., Figure 2 
A Anti-SARS-CoV S (Alexa Fluor 647)

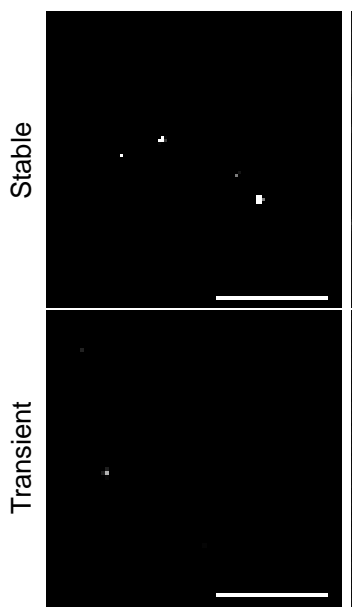

Anti-VSV M

(Alexa Fluor 488)

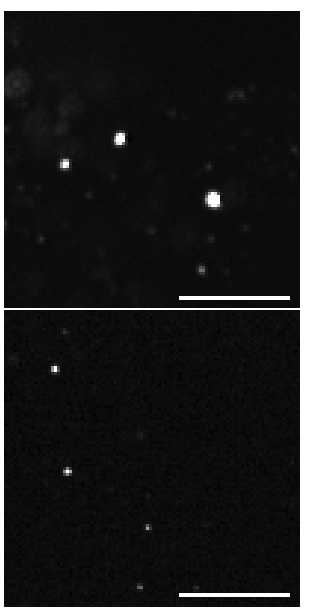

Merged

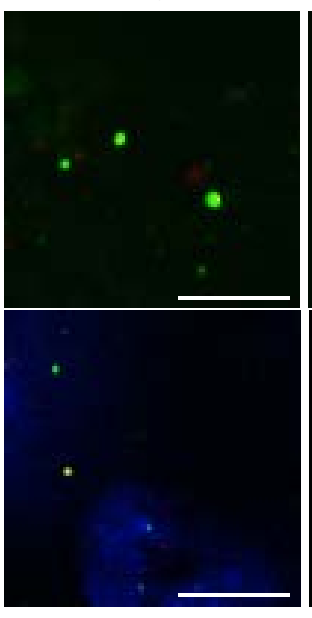

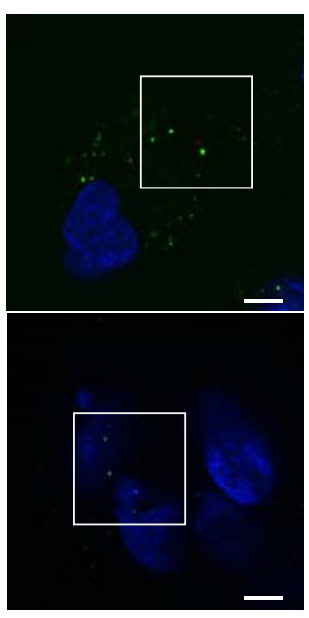

B

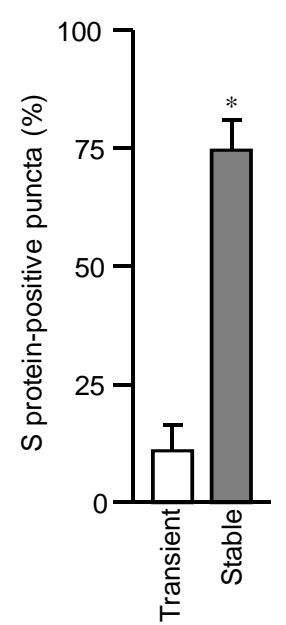

C

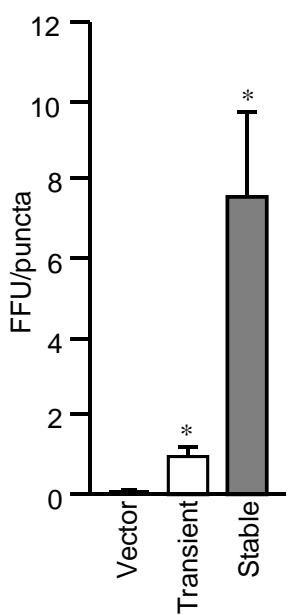


bioRxiv preprint dqj: https://doi.org/10.1101/2021.07.30.454063; this version posted July 30, 2021. The copyright holder for this preprint (which was not certified by pserablieview) is the author/funder All hrights reserved. Noomuse allowed without permission.

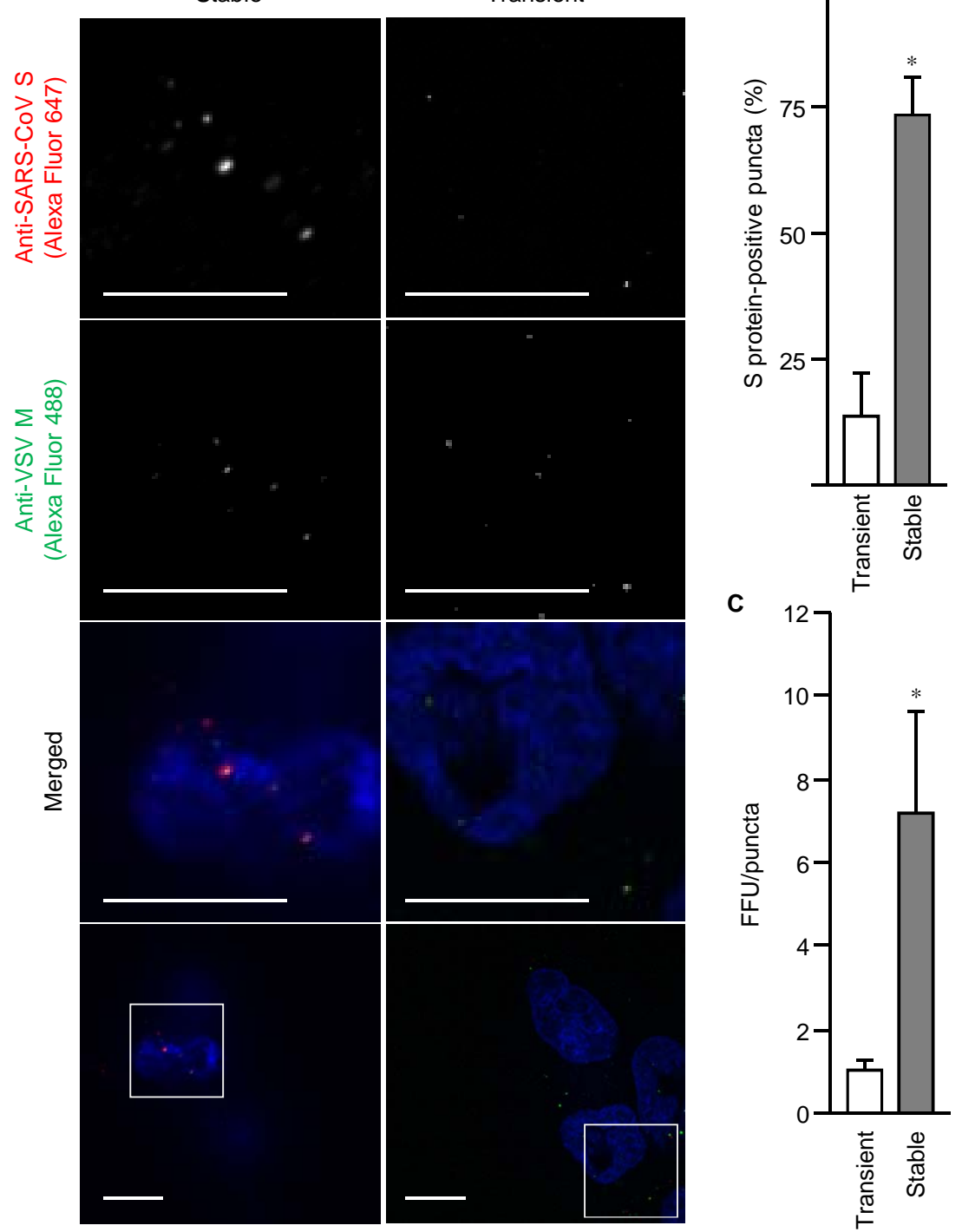

